\title{
Portfolio Optimization Problem with Non-identical Variances of Asset Returns using Statistical Mechanical Informatics
}

\author{
Takashi Shinzatd* \\ Mori Arinori Center for Higher Education and Global Mobility, Hitotsubashi University, Tokyo, 1868601, Japan.
}

(Dated: September 12, 2018)

\begin{abstract}
The portfolio optimization problem in which the variances of the return rates of assets are not identical is analyzed in this paper using the methodology of statistical mechanical informatics, specifically, replica analysis. We define two characteristic quantities of an optimal portfolio, namely, minimal investment risk and concentrated investment level, in order to solve the portfolio optimization problem and analytically determine their asymptotical behaviors using replica analysis. Moreover, numerical experiments were performed, and a comparison between the results of our simulation and those obtained via replica analysis validated our proposed method.
\end{abstract}

PACS number(s): 89.65.Gh, 89.90.+n, 02.50.-r

\section{INTRODUCTION}

Investment is an economic activity in which one pays a cost according to the reward that can be expected in the future 1, 2]. In 1952, in the field of investment science, Markowitz introduced the portfolio optimization problem and constructed as a framework of investment management portfolios with relatively low risk that do not rely on either experience or intuition [3, 4]. More concretely, the case of investing a finite budget into several assets appropriately is considered, for instance, Markowitz proposed the investment behavior policy that a portfolio that minimizes the expectation of investment risk, defined as the variance of the expected returns under the sum of expected returns being constant, be regarded as an optimal portfolio and analyzed what investment strategy could realize such an optimal portfolio. Konno et al. proposed the absolute deviation model, in which the sum of the absolute value of the expected return over all periods is regarded as the investment risk, rather than the sum of the square of the expected return, and indicated that the portfolio of minimal expected investment risk of the absolute deviation model is consistent with the portfolio of minimal expected investment risk of the mean-variance model which was introduced by Markowitz [5]. Rockafellar et al. introduced the expected shortfall model that assesses which portfolios have an investment risk which is not below a confidence level, taking into account the risk of expected returns [6].

In previous decades, portfolio optimization problems which could not be easily solved using the analytical approach developed in operations research have been solved using statistical mechanical informatics $[-10]$. For instance, Ciliberti et al. analyzed the typical behavior of the minimal investment risks of the absolute deviation model and the expected shortfall model which were derived in the limit of absolute zero temperature using replica analysis developed in statistical mechanical

\footnotetext{
*takashi.shinzato@r.hit-u.ac.jp
}

informatics[7]. Furthermore, Pafka et al. proposed a method for excluding noise from the variance-covariance matrix of asset returns using random matrix theory which has been improved in econophysics, evaluated the concentrated investment level using the Marčenko-Pastur law, and compared the estimated returns and the actual returns [8]. Although a computation proportional to the cube of the number of assets is needed in order to produce straightforwardly the inverse of the variance-covariance matrix in the case of the mean-variance model, Shinzato et al. developed a faster algorithm in order to derive the optimal portfolio for the risk minimization problem with a budget constraint by using belief propagation from statistical mechanical informatics [9]. Shinzato also indicated that the minimal investment risk and its concentrated investment level satisfy the self-averaging property by using replica analysis, which supports the theoretical approach of Ciliberti et al. [10].

Such previous works partly discuss the potential of the optimal portfolio in their investment systems by using analytical approaches developed in cross-disciplinary research involving the portfolio optimization problem and statistical mechanical informatics. However, these works do not reveal their systems' full potential. In practice, these works primarily consider the portfolio optimization problem under the assumption that the returns of assets are independently and identically distributed or that the return of each asset is independently distributed and is not identical but that the variances of return rates of the assets are the same, for simplicity. This assumption ignores the fact that there exist risk-free assets and risky assets, such as national government bonds and corporate bonds of small and medium-sized firms, in actual securities market. Since there exist a wide variety of types of assets, previous methods [7-10] are insufficient for handling investment management in an actual securities market.

Therefore, in this paper, we analyze the portfolio optimization problem, especially, the mean-variance model, in which the return rates of assets in the market are independently distributed and have different variances, and assess the typical behaviors of the minimal invest- 
ment risk per asset and its concentrated investment level using replica analysis. Numerical experiments using a belief propagation algorithm [9] were performed and a comparison between the results of our simulation and those obtained via replica analysis validated our proposed method.

\section{MATERIALS AND METHODS}

\section{A. Mean-variance model}

In this subsection, we concretely introduce one of the most representative risk management models applied to the portfolio optimization problem, the mean-variance model. First, as the securities market handled in the present paper, we assume that the investor can invest in $N$ assets without constraints on short selling and the investment ratio of the portfolio invested in asset $i(=1, \cdots, N)$ is represented as $w_{i} \in \mathbf{R}$ and the total portfolio is expressed as the $N$-dimensional vector $\vec{w}=\left(w_{1}, \cdots, w_{N}\right)^{\mathrm{T}} \in \mathbf{R}^{N}$, where notation $\mathrm{T}$ herein means the transposition of a vector or matrix. Furthermore, we assume here that the distribution of the return rates of $N$ assets across $p$ scenarios is known, and the return rate of asset $i$ in scenario $\mu(=1, \cdots, p)$ is $x_{i \mu}^{\prime}$, where the expectation of the return rate of asset $i$ is represented as $r_{i}=E\left[x_{i \mu}^{\prime}\right]$, which is known. From this, the return rates of all assets in $p$ scenarios are known at time of investing, the investment risk of the mean-variance model $\mathcal{H}(\vec{w} \mid X)$ of portfolio $\vec{w}$ is defined as follows:

$$
\begin{aligned}
\mathcal{H}(\vec{w} \mid X) & =\frac{1}{2 N} \sum_{\mu=1}^{p}\left(\sum_{i=1}^{N} x_{i \mu}^{\prime} w_{i}-\sum_{i=1}^{N} r_{i} w_{i}\right)^{2} \\
& =\frac{1}{2} \sum_{\mu=1}^{p}\left(\frac{1}{\sqrt{N}} \sum_{i=1}^{N} x_{i \mu} w_{i}\right)^{2}
\end{aligned}
$$

where the normalized asset return rate $x_{i \mu}=x_{i \mu}^{\prime}-r_{i}$ has the mean normalized to zero for simplicity of discussion below (hereafter, we use $x_{i \mu}$ not $x_{i \mu}^{\prime}$ where this does not cause confusion in our discussion). In addition, the return rectangular matrix $X=\left\{\frac{x_{i \mu}}{\sqrt{N}}\right\} \in \mathcal{M}_{N \times p}$ whose components are normalized return rates is used. Next, as a budget constraint, the sum of investment ratios

$$
\sum_{i=1}^{N} w_{i}=N
$$

is employed. In this work, as in cross-disciplinary research fields involving the portfolio optimization problem and statistical mechanical informatics, so as to simplify our discussion, we include a budget constraint only, that is, we do not include an expected returns constraint. Note that the budget constraint in previous works in the operations research field is $\sum_{i=1}^{N} w_{i}=1$, not Eq (2). We explain our difference in choice below.
The mean-variance model handled in the present paper, then, is reformulated as an optimization problem in which we minimize the investment risk $\mathcal{H}(\vec{w} \mid X)$ given in Eq (11) with respect to portfolio $\vec{w}$ under the budget constraint Eq (2). In general, when the rank of the return matrix $X=\left\{\frac{x_{i \mu}}{\sqrt{N}}\right\} \in \mathcal{M}_{N \times p}$ is $N$, in brief, when $N<p$, it can be shown that the optimal portfolio is uniquely determined as follows:

$$
\vec{w}=\frac{N J^{-1} \vec{e}}{\vec{e}^{T} J^{-1} \vec{e}}
$$

where the constant vector is $\vec{e}=(1, \cdots, 1)^{\mathrm{T}} \in \mathbf{R}^{N}$ and the variance-covariance matrix $J=\left\{J_{i j}\right\}=X X^{\mathrm{T}} \in$ $\mathcal{M}_{N \times N}$, gives the covariance between asset $i$ and asset $j$,

$$
J_{i j}=\frac{1}{N} \sum_{\mu=1}^{p} x_{i \mu} x_{j \mu} .
$$

Note that $J^{-1}$, the inverse matrix of variance-covariance matrix $J$, exists when $N<p$, since the ranks of the return matrix $X$ and the variance-covariance matrix $J$ are assumed to be $N$, and the optimal portfolio is uniquely determined. However, when $N>p$, since $J$ is not assumed to be invertible, the optimal portfolio is not uniquely determined. Hereafter, we limit our discussion to the $N<p$ case.

We will consider the following two statistics as feature quantities which can characterize the potential of a portfolio:

$$
\begin{aligned}
\varepsilon & =\frac{1}{N} \mathcal{H}(\vec{w} \mid X), \\
q_{w} & =\frac{1}{N} \sum_{i=1}^{N} w_{i}^{2},
\end{aligned}
$$

where $\varepsilon$ defined by Eq (5) is the investment risk per asset and $q_{w}$ defined by $\mathrm{Eq}$ (6) is the concentrated investment level. The nature of $\varepsilon$ can be intuitively comprehended; however, since the properties of $q_{w}$ have not been sufficiently discussed in the literature and $q_{w}$ is not widely used in operations research, the properties of concentrated investment level will be briefly explained here. For two typically considered investment strategies, the equipartition investment strategy (EIS) and the concentrated investment strategy (CIS), the portfolios are represented by $\vec{w}^{\mathrm{EIS}}=(1,1, \cdots, 1)^{\mathrm{T}} \in \mathbf{R}^{N}$ and $\vec{w}^{\text {CIS }}=(N, 0, \cdots, 0)^{\mathrm{T}} \in \mathbf{R}^{N}$, respectively. In the CIS case, we assume that the total budget is invested in asset 1 to simply our discussion. Then, the concentrated investment levels of the investment strategies are $q_{w}^{\text {EIS }}=1$ and $q_{w}^{\mathrm{CIS}}=N$, respectively, and we see that $q_{w}^{\mathrm{EIS}}<q_{w}^{\mathrm{CIS}}$. That is, when the concentrated investment level of an investment strategy is small, that investment strategy is regarded as the equipartition investment strategy, and otherwise the investment strategy is treated as the concentrated investment strategy. From this, it is clear that the concentrated investment level can be used as a measure of the degree of investment diversification. 
Three points should be noted at this point. First, expanding on the above discussion, from the definition of the concentrated investment level in Eq (6), $q_{w} \geq 1$ is satisfied. This follows from using the mean square of the portfolio, $\frac{1}{N} \sum_{i=1}^{N} w_{i}^{2}$, and the mean of the portfolio, $\frac{1}{N} \sum_{i=1}^{N} w_{i}$, as follows:

$$
\begin{aligned}
q_{w}-1 & =\frac{1}{N} \sum_{i=1}^{N} w_{i}^{2}-\left(\frac{1}{N} \sum_{i=1}^{N} w_{i}\right)^{2} \\
& =\frac{1}{N} \sum_{i=1}^{N}\left(w_{i}-\frac{1}{N} \sum_{i=1}^{N} w_{i}\right)^{2} .
\end{aligned}
$$

This bound implies that the concentrated investment level in the equipartition investment strategy is a lower bound on the possible concentrated investment level, while the concentrated investment level in the concentrated investment strategy is increasing with increasing concentration level of investing into specific assets.

Second, related to the first point, if the definitions of the concentrated investment level and the budget constraint follow the formulation widely used in operations research, for instance, $q_{w}^{(1)}=\sum_{i=1}^{N}\left(w_{i}^{(1)}\right)^{2}$ and $\sum_{i=1}^{N} w_{i}^{(1)}=1$, then concentrated investment level $q_{w}^{(1)}=$ $\sum_{i=1}^{N}\left(w_{i}^{(1)}\right)^{2}$ does not easily provide statistical interpretations like that in Eq (77). In addition, under the two budget constraints $\sum_{i=1}^{N} w_{i}^{(1)}=1$ and $\sum_{i=1}^{N} w_{i}^{(N)}=N$, optimal portfolios in Eq (11) are denoted as $\vec{w}^{(1)}$ and $\vec{w}^{(N)}$, respectively, and the portfolio ratios of asset $i$ to asset $j$ for the two optimal portfolios are consistent with each other, $w_{i}^{(1)} / w_{j}^{(1)}=w_{i}^{(N)} / w_{j}^{(N)}$, since $\vec{w}^{(N)}=N \vec{w}^{(1)}$. Because of this agreement, we choose to use the budget constraint Eq (2), since the concentrated investment level alone is sufficient for statistical interpretations, unlike the budget constraint widely used in operations research.

Lastly, we substitute the optimal portfolio given in Eq (3) into $\varepsilon$ in $\mathrm{Eq}$ (5) and $q_{w}$ in Eq (6), which gives

$$
\begin{aligned}
\varepsilon & =\frac{1}{2\left(\frac{1}{N} \vec{e}^{\mathrm{T}} J^{-1} \vec{e}\right)}, \\
q_{w} & =\frac{\frac{1}{N} \vec{e}^{\mathrm{T}} J^{-2} \vec{e}}{\left(\frac{1}{N} \vec{e}^{\mathrm{T}} J^{-1} \vec{e}\right)^{2}} .
\end{aligned}
$$

However, the computation of the inverse of the variancecovariance matrix is $O\left(N^{3}\right)$, while the number of assets $N$ is large, and so $\frac{1}{N} \vec{e}^{T} J^{-1} \vec{e}$ and $\frac{1}{N} \vec{e}^{T} J^{-2} \vec{e}$ are not easy to calculate in practical time. Hereafter, we call $\varepsilon$ and $q_{w}$ of the optimal portfolio given in Eq (3) the minimal investment risk (per asset) and the concentrated investment level of the optimal portfolio, respectively, and we focus our analyze on these two characteristic quantities in order to evaluate the potential of an investment system.

With respect to the problem of computation size of determining the optimal portfolio, for the case that the return rates of assets are independently and identically distributed with mean and variance 0 and 1 , respectively, $\varepsilon$ and $q_{w}$ have already been analyzed theoretically and numerically using replica analysis in previous works [9, 10], in which the follow analytic formulations are reported:

$$
\begin{aligned}
\varepsilon & =\frac{\alpha-1}{2}, \\
q_{w} & =\frac{\alpha}{\alpha-1},
\end{aligned}
$$

where we use the ratio of the number of scenarios $p$ to the number of assets $N$, that is, the scenario rate $\alpha=p / N$. These formulations handle the $\alpha>1$ case $(p>N)$ since in this case the optimal portfolio is uniquely determined.

In general, in an investment system, the return rates of assets in a securities market are not always independently and identically distributed, but rather can be correlated with each other and have different variances. Therefore, we need to implement asset management that appropriately combines assets in the securities market, including risk-free assets such as national government bonds and risky assets such as corporate bonds of small and medium-sized firms, unlike the approaches reported in previous works, which are not sufficient to handle the portfolio optimization problem when the variances of the return rates of assets are not identical.

Thus, our aim in this work is to analyze the optimal portfolio of the portfolio optimization problem with non-identical variances of the return rates of assets. For instance, investment assets are constructed to comprise risk-free assets and comparatively risky assets, since one can comparatively well characterize the nature of the actual market in this situation. An additional aim is to develop a replica approach to solve this problem and to examine the typical behaviors of the minimal investment risk and the concentrated investment level of the optimal solution in a way similar to that used in previous works. Specifically, in this paper, we assume that the return rates of assets are uncorrelated with one another and write the variance of asset $i, E_{X}\left[x_{i \mu}^{2}\right]-\left(E_{X}\left[x_{i \mu}\right]\right)^{2}=$ $E_{X}\left[x_{i \mu}^{2}\right]$, as

$$
E_{X}\left[x_{i \mu}^{2}\right]=s_{i},
$$

for simplicity. In addition, we assume that the $l$ th moment of the return rate, $E_{X}\left[x_{i \mu}^{l}\right]$, does not depend on the number of assets and/or is finite, since it is known that the return rates of assets are not directly influenced by the asset size of the securities market.

\section{B. Boltzmann distribution}

In this subsection, we reformulate the previously stated portfolio optimization problem using the Boltzmann distribution in the framework of probabilistic inference, similar to as was used in previous works, in order to apply seamlessly the replica analysis approach. Using the literature of probabilistic inference, the posteriori probability 
of portfolio $\vec{w}$ is defined as follows:

$$
P(\vec{w} \mid X)=\frac{P_{0}(\vec{w}) e^{-\beta \mathcal{H}(\vec{w} \mid X)}}{Z(X)},
$$

where the denominator in Eq (13) is defined as

$$
Z(X)=\int_{-\infty}^{\infty} d \vec{w} P_{0}(\vec{w}) e^{-\beta \mathcal{H}(\vec{w} \mid X)},
$$

which is called the partition function and plays a central role in the analysis of this paper. Moreover, the prior probability $P_{0}(\vec{w})$ is related in the budget constraint in Eq (2) and is exponentially described as $P_{0}(\vec{w}) \propto$ $\frac{1}{(2 \pi)^{\frac{N}{2}}} e^{k\left(\sum_{k=1}^{N} w_{i}-N\right)}$, where auxiliary variable $k$ is chosen to satisfy $\mathrm{Eq}(2)$. In addition, $e^{-\beta \mathcal{H}(\vec{w})}$ is a likelihood function or Boltzmann factor with inverse temperature $\beta(>0)$, which is the control parameter in deriving the optimal solution.

In practice, from the definition of the posteriori probability given in $\mathrm{Eq}(13)$, because Boltzmann factor $e^{-\beta \mathcal{H}}$ is a monotonic nonincreasing function with respect to $\mathcal{H}$, the maximum a posteriori estimate is consistent with the optimal portfolio of the portfolio optimization problem. In addition, posteriori probability $P(\vec{w} \mid X)$ in a neighborhood of the maximum a posteriori estimate (the optimal portfolio of the portfolio optimization problem) increases as inverse temperature $\beta$ becomes large, whereas posteriori probability $P(\vec{w} \mid X)$ outside the neighborhood decreases to 0 as $\beta$ increases. In the limit of large $\beta$, the expectation of $\mathcal{H}(\vec{w} \mid X), E_{\vec{w}}[\mathcal{H}(\vec{w} \mid X)]=$ $\int_{-\infty}^{\infty} d \vec{w} P(\vec{w} \mid X) \mathcal{H}(\vec{w} \mid X)$, approaches

$$
\lim _{\beta \rightarrow \infty} E_{\vec{w}}[\mathcal{H}(\vec{w} \mid X)]=\mathcal{H}\left(\vec{w}^{*} \mid X\right)
$$

where $\vec{w}^{*}$ is the maximum a posteriori estimate. Moreover, this is related to the Boltzmann distribution as follows:

$$
\begin{aligned}
E_{\vec{w}}[\mathcal{H}(\vec{w} \mid X)] & =\int_{-\infty}^{\infty} d \vec{w} P(\vec{w}) \mathcal{H}(\vec{w} \mid X) \\
& =-\frac{\partial}{\partial \beta} \log Z(X),
\end{aligned}
$$

which is known as the identical equation. That is, from the properties in Eq (15) and Eq (16), the minimal investment risk per asset $\varepsilon$ is estimated using the following identical equation:

$$
\begin{aligned}
\varepsilon & =\frac{1}{N} \mathcal{H}\left(\vec{w}^{*} \mid X\right) \\
& =\lim _{\beta \rightarrow \infty}\left(-\frac{\partial}{\partial \beta} \frac{1}{N} \log Z(X)\right) .
\end{aligned}
$$

\section{Replica analysis}

In order to examine the potential investment risk in our handled investment system, we need to evaluate the expectation of minimal investment risk; however, it is well known that it is difficult to directly assess the expectation of $\varepsilon$ defined in Eq (8) and/or the expectation of $\varepsilon$ defined in Eq (17). Therefore, we will resolve this difficulty by using replica analysis in a way similar to that in previous works. Specifically, since the expectation of $\varepsilon$ defined in Eq (8) is consistent with the expectation of $\varepsilon$ defined in Eq (17), we derive $\varepsilon=\lim _{\beta \rightarrow \infty} \frac{1}{N} E_{X}\left[E_{\vec{w}}[\mathcal{H}(\vec{w} \mid X)]\right]$ from $\frac{1}{N} E_{X}[\log Z(X)]$, although, unlike the specific model considered here, in general, directly averaging the logarithm of a random variable and/or statistic $Z(X)$ is not easy. Therefore, we make use of either a replica trick,

$$
\log Z(X)=\lim _{n \rightarrow 0} \frac{Z^{n}(X)-1}{n},
$$

or the expectation description of the replica trick [13],

$$
E_{X}[\log Z(X)]=\lim _{n \rightarrow 0}\left\{\begin{array}{l}
\frac{E_{X}\left[Z^{n}(X)\right]-1}{n} \\
\frac{\log E_{X}\left[Z^{n}(X)\right]}{n \log E_{X}^{n}\left[Z^{n}(X)\right]} \\
\partial n
\end{array} .\right.
$$

in order to analyze the expected value of the logarithm of the partition function. Thus, it is necessary to analytically assess $E_{X}\left[Z^{n}(X)\right]$ in the replica trick. We will need the following functions in order to perform this assessment using replica analysis:

$$
\begin{aligned}
\psi(n) & =\lim _{N \rightarrow \infty} \frac{1}{N} \log E_{X}\left[Z^{n}(X)\right], \\
\phi & =\lim _{N \rightarrow \infty} \frac{1}{N} E_{X}[\log Z(X)] .
\end{aligned}
$$

From these definitions, obviously,

$$
\phi=\lim _{n \rightarrow 0} \frac{\partial \psi(n)}{\partial n},
$$

holds and the minimal investment risk per asset $\varepsilon$ is calculated as follows:

$$
\varepsilon=\lim _{\beta \rightarrow \infty}\left(-\frac{\partial \phi}{\partial \beta}\right),
$$

where hereafter the expectation of the minimal investment risk per asset is also written simply as $\varepsilon$ for convenience.

In replica analysis, our main goal is to analyze $\psi(n)$ defined in Eq (20) in order to examine the typical behaviors of these statistics which characterize the investment systems. Determining these behaviors is easier than directly evaluating the expectation of the logarithm of the partition function; however, it is well known that it is hard to directly evaluate $\psi(n)(n \in \mathbf{R})$, except for at specific values of $n$. On the other hand, since we first are possible to implement the configurational average of $Z^{n}(X)$ over the return rate matrix $X$ with respect to some $n \in \mathbf{N}$ using polynomial expansion of the partition function, we consider $E_{X}\left[Z^{n}(X)\right]$ only for $n \in \mathbf{N}$. It is hoped that the results for $n \in \mathbf{N}$ will provide cues for determining the moment of the partition function at $n \in \mathbf{R}$. Thus, 
for $n \in \mathbf{N}$, we will use the expansion

$$
\begin{aligned}
E_{X}\left[Z^{n}(X)\right]= & \int_{-\infty}^{\infty} \prod_{a=1}^{n} d \vec{w}_{a} P_{0}\left(\vec{w}_{a}\right) \\
& E_{X}\left[\exp \left(-\beta \sum_{a=1}^{n} \mathcal{H}\left(\vec{w}_{a} \mid X\right)\right)\right]
\end{aligned}
$$

where $\vec{w}_{a}=\left(w_{1 a}, w_{2 a}, \cdots, w_{N a}\right)^{\mathrm{T}} \in \mathbf{R}^{N}, a=1,2, \cdots, n$, is as defined previously above and $a$ is the replica index. Moreover, since the return rate is independently drawn from a distribution with mean and variance $E_{X}\left[x_{i \mu}\right]=$ 0 and $E_{X}\left[x_{i \mu}^{2}\right]-\left(E_{X}\left[x_{i \mu}\right]\right)^{2}=s_{i}$, respectively, and $\lim _{N \rightarrow \infty} N^{-\frac{l}{2}} E_{X}\left[\left(x_{i \mu}\right)^{l}\right]=0,(l=1,2,3, \cdots)$, it is easy to implement the configurational averaging with the return distribution in our replica analysis in a way similar to that used in previous works [7, 10].

In order to calculate the right-hand side of Eq (24), let us here define two types of order parameters as follows:

$$
\begin{aligned}
q_{w a b} & =\frac{1}{N} \sum_{i=1}^{N} w_{i a} w_{i b}, \\
q_{s a b} & =\frac{1}{N} \sum_{i=1}^{N} w_{i a} w_{i b} s_{i},
\end{aligned}
$$

where $a, b=1,2, \cdots, n$ are replica indices and $\tilde{q}_{w a b}$ and $\tilde{q}_{s a b}$ are defined as conjugate auxiliary variables of $q_{w a b}$ and $q_{s a b}$. Also, we define sets $Q_{w}=\left\{q_{w a b}\right\}, \tilde{Q}_{w}=$ $\left\{\tilde{q}_{w a b}\right\}, Q_{s}=\left\{q_{s a b}\right\}, \tilde{Q}_{s}=\left\{\tilde{q}_{s a b}\right\} \in \mathcal{M}_{n \times n}$. Note that for the case $a=b, \mathrm{Eq}$ (25) corresponds to the concentrated investment level defined in Eq (4) and that we derive the order parameter $q_{s a b}$ and its conjugate auxiliary parameter $\tilde{q}_{s a b}$ as the novel variables in order to handle the non-identical case (only $q_{w a b}, \tilde{q}_{w a b}$, and $k_{a}$ are used in our previous work[10]).

Using the above variable definitions, Eq (24) can be expanded as follows:

$$
\begin{aligned}
\psi(n)= & \operatorname{Extr}_{\Theta}\left\{-\vec{e}^{\mathrm{T}} \vec{k}+\frac{1}{2} \vec{k}^{\mathrm{T}}\left\langle\left(\tilde{Q}_{w}+s \tilde{Q}_{s}\right)^{-1}\right\rangle \vec{k}\right. \\
& +\frac{1}{2} \operatorname{Tr} Q_{s} \tilde{Q}_{s}+\frac{1}{2} \operatorname{Tr} Q_{w} \tilde{Q}_{w}-\frac{\alpha}{2} \log \operatorname{det}\left|I+\beta Q_{s}\right| \\
& \left.-\frac{1}{2}\left\langle\log \operatorname{det}\left|\tilde{Q}_{w}+s \tilde{Q}_{s}\right|\right\rangle\right\},
\end{aligned}
$$

where $\vec{e}=(1, \cdots, 1)^{\mathrm{T}} \in \mathbf{R}^{n}$ is a vector of constants, $\vec{k}=\left(k_{1}, \cdots, k_{n}\right)^{\mathrm{T}} \in \mathbf{R}^{n}$ is an auxiliary vector, $I \in \mathcal{M}_{n \times n}$ is the identity matrix, and $\Theta=\left\{\vec{k}, Q_{w}, Q_{s}, \tilde{Q}_{w}, \tilde{Q}_{s}\right\}$, in which $Q_{w}, Q_{s}, \tilde{Q}_{w}$, and $\tilde{Q}_{s}$ are as defined above. Furthermore, the notation

$$
\langle f(s)\rangle=\lim _{N \rightarrow \infty} \frac{1}{N} \sum_{i=1}^{N} f\left(s_{i}\right)
$$

is used and $\operatorname{Extr}_{\Theta} f(\Theta)$ means the extremum of the function $f(\Theta)$ with respect to $\Theta$. Moreover, $p$ and $N$ are taken to approach infinity in order to maintain a finite scenario ratio $\alpha=p / N \sim O(1)$. Therefore, the extrema of the right-hand side of Eq (27) with respect to the order parameters are as follows:

$$
\begin{aligned}
\frac{\partial \psi(n)}{\partial \vec{k}}= & -\vec{e}+\left\langle\left(\tilde{Q}_{w}+s \tilde{Q}_{s}\right)^{-1}\right\rangle \vec{k}=0 \\
\frac{\partial \psi(n)}{\partial Q_{w}}= & \frac{1}{2} \tilde{Q}_{w}=0 \\
\frac{\partial \psi(n)}{\partial Q_{s}}= & \frac{1}{2} \tilde{Q}_{s}-\frac{\alpha \beta}{2}\left(I+\beta Q_{s}\right)^{-1}=0 \\
\frac{\partial \psi(n)}{\partial \tilde{Q}_{w}}= & -\frac{1}{2}\left\langle\left(\tilde{Q}_{w}+s \tilde{Q}_{s}\right)^{-1} \vec{k} \vec{k}^{\mathrm{T}}\left(\tilde{Q}_{w}+s \tilde{Q}_{s}\right)^{-1}\right\rangle \\
& -\frac{1}{2}\left\langle\left(\tilde{Q}_{w}+s \tilde{Q}_{s}\right)^{-1}\right\rangle+\frac{1}{2} Q_{w}=0 \\
\frac{\partial \psi(n)}{\partial \tilde{Q}_{s}}= & -\frac{1}{2}\left\langle s\left(\tilde{Q}_{w}+s \tilde{Q}_{s}\right)^{-1} \vec{k} \vec{k}^{\mathrm{T}}\left(\tilde{Q}_{w}+s \tilde{Q}_{s}\right)^{-1}\right\rangle \\
& -\frac{1}{2}\left\langle s\left(\tilde{Q}_{w}+s \tilde{Q}_{s}\right)^{-1}\right\rangle+\frac{1}{2} Q_{s}=0
\end{aligned}
$$

From these, the extrema of $Q_{w}, Q_{s}, \tilde{Q}_{w}, \tilde{Q}_{s}$, and $\vec{k}$ are determined as follows:

$$
\begin{aligned}
Q_{w} & =\frac{\left\langle s^{-1}\right\rangle}{\beta(\alpha-1)} I+\left(\frac{\left\langle s^{-2}\right\rangle}{\left\langle s^{-1}\right\rangle^{2}}+\frac{1}{\alpha-1}\right) D, \\
Q_{s} & =\frac{1}{\beta(\alpha-1)} I+\frac{\alpha}{\left\langle s^{-1}\right\rangle(\alpha-1)} D, \\
\tilde{Q}_{w} & =0 \\
\tilde{Q}_{s} & =\beta(\alpha-1) I-\frac{\beta^{2}(\alpha-1)}{\left\langle s^{-1}\right\rangle+n \beta} D, \\
\vec{k} & =\frac{\beta(\alpha-1)}{\left\langle s^{-1}\right\rangle+n \beta} \vec{e},
\end{aligned}
$$

where $D=\vec{e} \vec{e}^{T} \in \mathcal{M}_{n \times n}$ is a square matrix with all components 1 . Note that we do not require the ansatz of the replica symmetry solution, just as in our previous work [10].

Substituting these results into the right-hand side of Eq (27), we analytically obtain

$$
\begin{aligned}
\psi(n)= & \frac{n}{2}-\frac{n}{2}\langle\log s\rangle-\frac{n \alpha}{2} \log \alpha+\frac{n(\alpha-1)}{2} \log (\alpha-1) \\
& -\frac{n}{2} \log \beta-\frac{\alpha-1}{2} \log \left(1+n \frac{\beta}{\left\langle s^{-1}\right\rangle}\right)
\end{aligned}
$$

where $\psi(0)=0$ is satisfied because $Z^{0}(X)=1$. Furthermore, although our analysis required the assumption $n \in \mathbf{N}$, the description of $\psi(n)$ given in Eq (39) can be applied more generally. Namely, we assume that Eq (39) has an analytic continuation to $n \in \mathbf{R}$ and substitute it into Eq (22), giving

$$
\begin{aligned}
\phi= & \frac{1}{2}-\frac{1}{2}\langle\log s\rangle-\frac{\alpha}{2} \log \alpha+\frac{\alpha-1}{2} \log (\alpha-1) \\
& -\frac{1}{2} \log \beta-\frac{\beta(\alpha-1)}{2\left\langle s^{-1}\right\rangle} .
\end{aligned}
$$


Furthermore, the minimal investment risk per asset $\varepsilon$ is obtained from Eq (23) as follows:

$$
\varepsilon=\frac{\alpha-1}{2\left\langle s^{-1}\right\rangle} .
$$

In a similar way, since concentrated investment level $q_{w}$ is evaluated by using $a=b$ in Eq (25), from the diagonal of $Q_{w}$ in Eq (34),

$$
q_{w}=\frac{\left\langle s^{-2}\right\rangle}{\left\langle s^{-1}\right\rangle^{2}}+\frac{1}{\alpha-1}
$$

can be analytically evaluated where the concentrated investment level of the optimal portfolio is derived from $q_{w}$ in the limit of sufficiently large $\beta$.

One last point should be noted here. It remains an open problem whether the result for the $n \in \mathbf{N}$ case extends to $n \in \mathbf{R}$ as an analytic continuation. Furthermore, we need to verify the effectiveness of our assumption, so we will compare the findings of our proposed method with those of numerical experiments below.

\section{Interpretation of our findings of two feature quantities}

Before the verification of the effectiveness of our assumption and our proposed approach by using numerical experiments, we give the interpretation of the results of our replica analysis.

a. (a) Two feature quantities depend only on the variance of the return rate distribution From Eq (41) and $\mathrm{Eq}$ (42), it is clear that these statistics do not depend on the other details of the return rate distribution besides the variances of the return rates of the assets. Suppose one rescales the return rate, such as in a stock split, with respect to a scaling coefficient of return $\sqrt{\gamma}$, that is, the rescaled return rate $\bar{x}_{i \mu}=\sqrt{\gamma} x_{i \mu}$ is defined from the original return rate $x_{i \mu}$. Then $E_{X}\left[\bar{x}_{i \mu}\right]=0$ and $E_{X}\left[\bar{x}_{i \mu}^{2}\right]=\gamma s_{i}$ are satisfied in the rescaled investment system, and the minimal investment risk is described by the following:

$$
\varepsilon(\gamma)=\lim _{N \rightarrow \infty} \frac{1}{2 N} \sum_{\mu=1}^{p}\left(\frac{1}{\sqrt{N}} \sum_{i=1}^{N} \bar{x}_{i \mu} w_{i}\right)^{2},
$$

and the concentrated investment level of the optimal portfolio is

$$
q_{w}(\gamma)=\lim _{N \rightarrow \infty} \frac{1}{N} \sum_{i=1}^{N} w_{i}^{2} .
$$

From replica analysis, these statistics are estimated as follows:

$$
\begin{aligned}
\varepsilon(\gamma) & =\frac{\gamma(\alpha-1)}{2\left\langle s^{-1}\right\rangle} \\
q_{w}(\gamma) & =\frac{\left\langle s^{-2}\right\rangle}{\left\langle s^{-1}\right\rangle^{2}}+\frac{1}{\alpha-1}
\end{aligned}
$$

Since the size of the portfolio of each asset is not changed by scaling coefficient $\sqrt{\gamma}$ under budget constraint Eq (2), the concentrated investment level is considered to be unchanged and the minimal investment risk changes by a factor of $\sqrt{\gamma}$.

b. (b) Comparison with the findings in previous works If the variances of the return rates of assets are identical, for instance, $s_{i}=1$, then $\left\langle s^{-1}\right\rangle=\left\langle s^{-2}\right\rangle=1$, and thus our results agree with Eq (10) and Eq (11) reported in previous works $[9,10$ ].

c. (c) Lower bound on concentrated investment level For the lower bound on the concentrated investment level, from the relation $\left\langle s^{-2}\right\rangle-\left\langle s^{-1}\right\rangle^{2}=$ $\left\langle\left(s^{-1}-\left\langle s^{-1}\right\rangle\right)^{2}\right\rangle \geq 0$, the relation $q_{w} \geq 1+\frac{1}{\alpha-1}=\frac{\alpha}{\alpha-1}$ can be obtained. Moreover, regarding the sharp lower bound, because $\left\langle s^{-2}\right\rangle-\left\langle s^{-1}\right\rangle^{2}=0$, the variances of the return rates of the assets are identical.

d. (d) Comparison with operations research findings In the present paper, we have found the optimal portfolio minimizing an investment risk $\mathcal{H}(\vec{w} \mid X)$ defined by a given return rate matrix, $X=\left\{\frac{x_{i \mu}}{\sqrt{N}}\right\} \in \mathcal{M}_{N \times p}$, and have determined the typical behaviors of two features of an optimal portfolio; in other words, we analyzed the quenched disorder system of this portfolio optimization problem discussed in the literature of statistical mechanical informatics [10]. For comparison, we will also analyze this optimal problem using the standard approach in operations research. In this approach, one first averages the investment risk $\mathcal{H}(\vec{w} \mid X)$ with the whole configuration of return rate matrix $X$. Next, one finds the optimal portfolio minimizing the expected investment risk $E_{X}[\mathcal{H}(\vec{w} \mid X)]$. The procedure of this approach is consistent with an annealed disorder system approach in the context of statistical mechanical informatics [10, 13].

Now, let us evaluate $\varepsilon$ and $q_{w}$ of the optimal portfolio in the annealed disorder system (the standard approach in operations research). From the statistical properties of the defined return rate, since $E_{X}\left[x_{i \mu}^{2}\right]=s_{i}$ and $E_{X}\left[x_{i \mu} x_{j \mu}\right]=E_{X}\left[x_{i \mu}\right] E_{X}\left[x_{j \mu}\right]=0,(i \neq j)$ are assumed above, the expected investment risk in the annealed disorder system is obtained as follows:

$$
E_{X}[\mathcal{H}(\vec{w} \mid X)]=\frac{\alpha}{2} \sum_{i=1}^{N} w_{i}^{2} s_{i},
$$

Furthermore, we find the optimal portfolio under the budget constraint in Eq (2), from which

$$
w_{i}^{\mathrm{OR}}=\frac{s_{i}^{-1}}{\left\langle s^{-1}\right\rangle},
$$

is obtained. This result implies that the portfolio is proportional to the inverse of the variance of the return rate of the asset. Finally, the concentrated investment level of the optimal portfolio minimizing the expected investment risk is briefly calculated as follows:

$$
q_{w}^{\mathrm{OR}}=\frac{\left\langle s^{-2}\right\rangle}{\left\langle s^{-1}\right\rangle^{2}} .
$$


This implies that the first term of the concentrated investment level in the quenched disorder system on the right-hand side of Eq (42) is caused by the annealed disorder system. As an interpretation of this relationship, since the return rates of assets are assumed to be independently distributed, there is no correlation between asset $i$ and asset $j$ in the expectation of the investment risk in Eq (47). In the other words, this investment system is equivalent to a securities market comprising $N$ assets which are uncorrelated with each other. However, in general, when deriving the solution which minimizes an investment risk defined by a given random return rate matrix $X$ in a quenched disorder system, the correlation terms ignored in the annealed disorder system cannot be ignored and so the second term on the right-hand side of Eq (42), $\frac{1}{\alpha-1}$, remains. Moreover, from another viewpoint, when $\alpha \simeq 1$, the optimal solution is close to the eigenvector of the minimal eigenvalue of $J=X X^{\mathrm{T}} \in \mathcal{M}_{N \times N}, \lambda_{\min }$. Since $\lambda_{\min }$ is close to 0 for $\alpha \simeq 1$ and $q_{w} \simeq \frac{1}{\lambda_{\min }^{2}}$, the concentrated investment level $q_{w}$ becomes infinity. Since the number of reference scenarios is relatively small, one interpretation is that an investor should concentrate investments in relatively riskless assets, based on the return rate table (like the previously mentioned concentrated investment strategy). On the other hand, when $\alpha \gg 1$, since the number of reference scenarios is large enough, the correlation term $x_{i \mu} x_{j \mu}$ is close to 0 relative to the autocorrelation term $x_{i \mu}^{2}$, the investment risk can be considered to be well approximated by the expected investment risk in the annealed disorder system, $E_{X}[\mathcal{H}(\vec{w} \mid X)]$. Furthermore, the behavior of the concentrated investment level is similar to that in an annealed disorder system.

Similarly, the minimal expected investment risk in the annealed disorder system is briefly calculated as follows:

$$
\varepsilon^{\mathrm{OR}}=\frac{\alpha}{2\left\langle s^{-1}\right\rangle} \text {. }
$$

If we compare the results of the quenched disorder system in Eq (41) with those of the annealed disorder system in Eq (50), it is clear that the effect of correlation between assets is captured by $-\frac{1}{2\left\langle s^{-1}\right\rangle}$.

\section{DISCUSSION}

In this section, we discuss the effectiveness of our proposed method by comparing its results with those from numerical experiments.

\section{A. Case 1: Two types of variances}

First, we consider here the portfolio optimization problem with two possible variances of the return rate; this problem relates to the case of risk-free and risky assets. Namely, we assign the variance of the return rate of asset $i, s_{i}$, as 1 with probability $r$ and as $\tilde{s}$ with probability $1-r$. Then, the expectation of $s^{-t},\left\langle s^{-t}\right\rangle$, is calculated as follows:

$$
\left\langle s^{-t}\right\rangle=r+(1-r) \tilde{s}^{-t}, \quad(t=1,2) .
$$

We implement numerical experiments using the following three numerical settings: (A) $\left\langle s^{-1}\right\rangle=3,\left\langle s^{-2}\right\rangle=30$, that is, $r=\frac{21}{25}, \tilde{s}=\frac{2}{27}$. (B) $\left\langle s^{-1}\right\rangle=4,\left\langle s^{-2}\right\rangle=30$, that is, $r=\frac{14}{23}, \tilde{s}=\frac{3}{26}$. (C) $\left\langle s^{-1}\right\rangle=5,\left\langle s^{-2}\right\rangle=30$, that is, $r=\frac{5}{21}, \tilde{s}=\frac{4}{25}$. Here, we solve for the portfolio which minimizes the investment risk $\mathcal{H}(\vec{w} \mid X)$ defined by a random return rate matrix $X$ whose entries are independently distributed with mean and variance 0 and $s_{i}$, respectively, by using the optimization algorithm based on the steepest descent method shown in Fig 1. This algorithm finds the extremum of Lagrange function $L(\vec{w}, \zeta)=\mathcal{H}(\vec{w} \mid X)+\zeta\left(N-\vec{e}^{\mathrm{T}} \vec{w}\right)$ with respect to portfolio $\vec{w}$ and auxiliary variable $\zeta$, where $\vec{e}=\{1,1, \cdots, 1\}^{\mathrm{T}} \in \mathbf{R}^{N}$.

The results of the algorithm based on the steepest descent method (symbols with error bars), the replica analysis (solid lines), and the standard approach in operations research (dashed lines) are shown in Figs. 2 and 3 In both figures, results appear in the order (A), (B), and (C) from top to bottom. More specifically, the symbols with error bars show the expectation of the minimal investment risk derived by numerical simulations, in which the number of assets is set as $N=1000$, and we randomly generate 100 return rate matrices and solve for the portfolio which minimizes the investment risk $\mathcal{H}(\vec{w} \mid X)$ by using the steepest descent method introduced in Fig. 1 .

Fortunately, these figures show that the typical behaviors of minimal investment risk derived by numerical experiment are in agreement with those derived by replica analysis. On the other hand, these figures also show that the standard approach in operations research is too difficult to analyze the potential of the optimization problem. Namely, it is found that our proposed method based on statistical mechanical informatics can assess the typical behaviors of the optimal solution minimizing this portfolio optimization problem without the difficulty of the standard approach.

\section{B. Case 2: Variance is distributed uniformly}

Next, we discuss the case that the variance of the return rate has a continuous uniform distribution. Namely, the range of the variance of the return rate of asset $i, s_{i}$, is given by $l_{s} \leq s_{i} \leq u_{s}$. Then $\left\langle s^{-1}\right\rangle=\frac{\log \frac{u_{s}}{l_{s}}}{u_{s}-l_{s}}$ and $\left\langle s^{-2}\right\rangle=\frac{1}{u_{s} l_{s}}$ are obtained and the minimal investment risk per asset and concentrated investment level are as follows:

$$
\begin{aligned}
\varepsilon & =\frac{\alpha-1}{2} \frac{u_{s}-l_{s}}{\log \frac{u_{s}}{l_{s}}} \\
q_{w} & =\frac{1}{u_{s} l_{s}}\left(\frac{u_{s}-l_{s}}{\log \frac{u_{s}}{l_{s}}}\right)^{2}+\frac{1}{\alpha-1} .
\end{aligned}
$$


Step 0: At $t=0$, set the initial state as $\vec{w}_{0}=\vec{e}$ and $\zeta_{0}=1$. Two small positive step sizes $\eta_{w}, \eta_{\zeta}>0$ are determined and a small positive constant number $\delta>0$ which is required in the stopping condition is set. In this study, $\eta_{w}=100 / N, \eta_{\zeta}=1 / N, \delta=10^{-6}$ are used.

Step 1: Using $\vec{w}_{t}, \zeta_{t}$, we determine $\vec{w}_{t+1}, \zeta_{t+1}$ as follows:

$$
\begin{aligned}
\vec{w}_{t+1} & =\vec{w}_{t}-\eta_{w}\left(X X^{\mathrm{T}} \vec{w}_{t}-\zeta_{t} \vec{e}\right), \\
\zeta_{t+1} & =\zeta_{t}+\eta_{\zeta}\left(N-\vec{e}^{\mathrm{T}} \vec{w}_{t}\right) .
\end{aligned}
$$

Step 2: If the L1 distance between $\vec{w}_{t+1}, \zeta_{t+1}$ and $\vec{w}_{t}, \zeta_{t}, \Delta=$ $\left|\zeta_{t+1}-\zeta_{t}\right|+\sum_{k=1}^{N}\left|w_{k, t+1}-w_{k, t}\right|$, is greater than $\delta$, then $t \rightarrow t+1$ and go to Step 1; otherwise, go to Step 3 .

Step 3: Return the results as the optimal portfolio (or its approximate value).

FIG. 1. Iterative algorithm for optimizing based on the steepest descent method.

In this case, we implement numerical simulations with the following three settings: $\left(\mathrm{A}^{\prime}\right) l_{s}=1, u_{s}=2$, (B') $l_{s}=1, u_{s}=3$, and ( $\left.\mathrm{C}^{\prime}\right) l_{s}=1, u_{s}=4$. For the numerical simulations, the number of assets is set as $N=1000$, and we randomly generate 100 return rate matrices and solve for the portfolio which minimizes the investment risk by using the steepest descent method introduced in Fig. 1. The results are shown in Figs. 4 and 5 . In both figures, results appear in the order $\left(\mathrm{A}^{\prime}\right),\left(\mathrm{B}^{\prime}\right)$, and $\left(\mathrm{C}^{\prime}\right)$ from bottom to top. Similar to in Case 1, the typical behaviors of the minimal investment risk derived by numerical experiments and the results derived by replica analysis are in good agreement, fortunately. On the other hand, these figures again show that the standard approach in operations research is too difficult to analyze the potential of the optimization problem. Namely, it is found again that our proposed method based on statistical mechanical informatics can assess the typical behaviors of the optimal solution minimizing this portfolio optimization problem without the difficulty of the standard approach.

\section{CONCLUSION}

In the present work, the portfolio optimization problem in which the variances of the return rates of assets are not identical, for instance, risk-free assets represented by national government bonds and risky assets represented by the corporate bonds of small and medium-sized firms, has been analyzed. The analysis in particular focused on two features, minimal investment risk and concentrated investment level, which were analyzed using replica analysis. Moreover, we discussed the potential of replica analysis developed in statistical mechanical informatics in resolving the portfolio optimization problem. The approach, which was developed by Shinzato et al. in previous works under the assumption of identical variances of the return rates of assets, is improved upon in the cur-

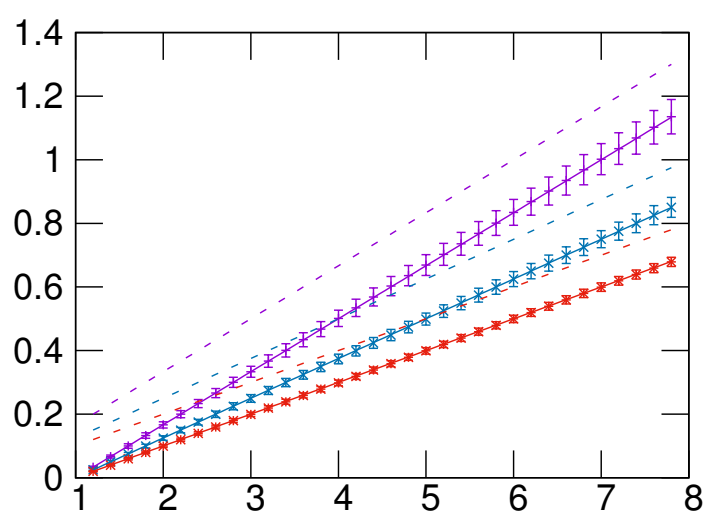

FIG. 2. Minimal investment risk per asset $\varepsilon$ versus scenario ratio $\alpha=p / N$ from a numerical experiment (symbols with error bars), our proposed approach (solid lines), and a standard operations research approach (dashed lines) for Case 1. Colors correspond to Case 1(A) (purple), Case 1(B) (blue), and Case 1(C) (red).

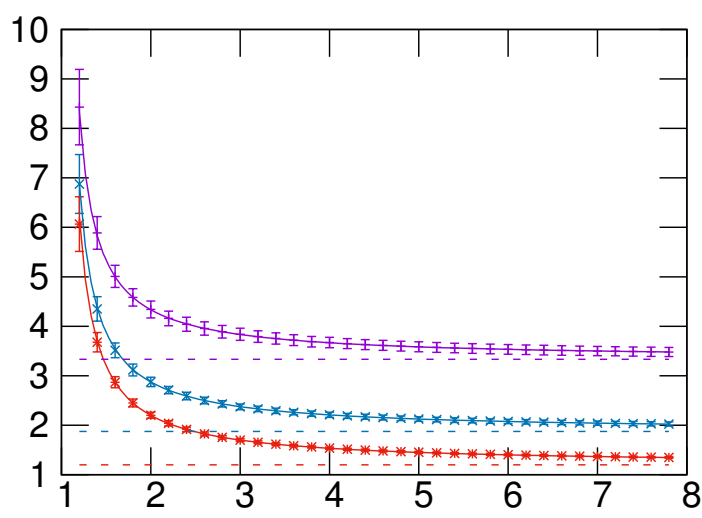

FIG. 3. Concentrated investment level of optima portfolio $q_{w}$ versus scenario ratio $\alpha=p / N$ from a numerical experiment (symbols with error bars), our proposed approach (solid lines), and a standard operations research approach (dashed lines) for Case 1. Colors correspond to Case 1(A) (purple), Case 1(B) (blue), and Case 1(C) (red).

rent work to allow it to solve the portfolio optimization problem with non-identical variances. With the improved method, the behavior of the optimal portfolio in the portfolio optimization problem can be derived using replica analysis. It was shown that if the scenario ratio is close to 1 , then the concentrated investment strategy gives the optimal portfolio, whereas if the scenario ratio is sufficiently large, then the equipartition investment strategy gives the optimal portfolio. In addition, we verified the effectiveness of our proposed method through numerical experiments.

In our future work, based on our assumption that the return rates of assets are independently distributed, for simplicity, though we developed the replica approach in previous works, for the actual securities market, we should consider correlation between assets, which the 
present work also ignored. Therefore, to analyze the nature of a real securities market, the minimal investment risk and concentrated investment level in the portfolio optimization problem in which returns are correlated with each other should be analyzed. Obviously, the proposed improvement in the present work does not allow analysis of a correlated investment system; for the correlated case, we need to develop an appropriate replica analysis approach.

\section{ACKNOWLEDGMENTS}

The author thanks I. Kaku and T. Mizuno for their fruitful comments. Also, the author is grateful to $\mathrm{R}$.

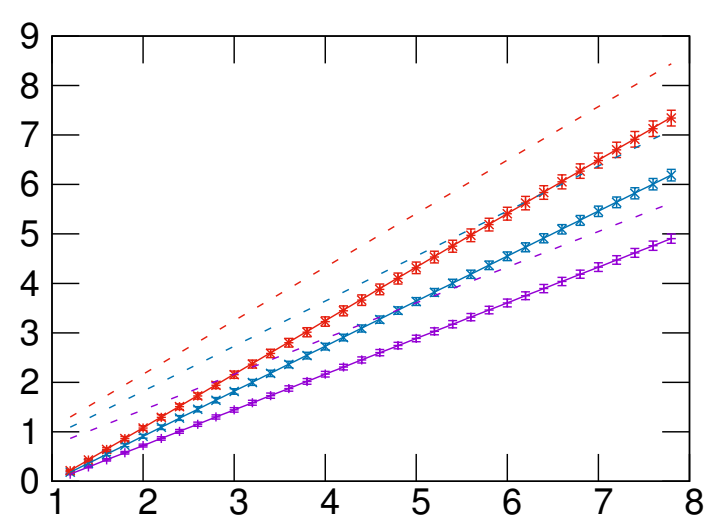

FIG. 4. Minimal investment risk per asset $\varepsilon$ versus scenario ratio $\alpha=p / N$ from a numerical experiment (symbols with error bars), our proposed approach (solid lines), and a standard operations research approach (dashed lines) for Case 2. Colors correspond to Case 2(A') (purple), Case 2(B') (blue), and Case 2(C') (red).

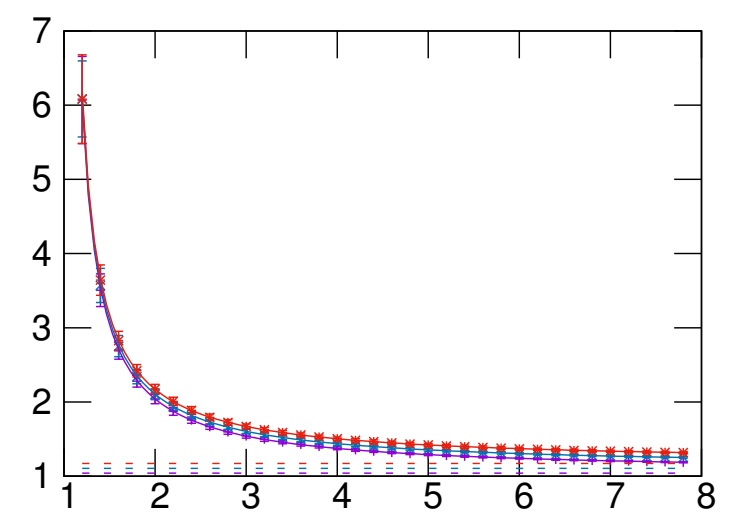

FIG. 5. Concentrated investment level of optima portfolio $q_{w}$ versus scenario ratio $\alpha=p / N$ from a numerical experiment (symbols with error bars), our proposed approach (solid lines), and a standard operations research approach (dashed lines) for Case 2. Colors correspond to Case 2(A') (purple), Case 2(B') (blue), and Case 2(C') (red).
Wakai and K. Kobayashi for valuable discussions. The work is partly supported by Grant-in-Aid Nos. 24710169 and 15K20999, President project for young scientists in Akita prefectural university, Research Project No. 50 in the National Institute of Informatics, Japan, Research Project No. 5 in Japan Institute of Life Insurance, Kyoto university institute of economic research foundation, Research Project No. 1414 in Zengin Foundation for Studies on Economics and Finance, Research Project No. 2068 in The institute of statistical mathematics, and Research Project No. 2 in Kampo foundation.

\section{APPENDIX}

\section{A. Replica analysis using replica symmetry ansatz}

In this appendix, using the replica symmetry ansatz, we analyze the behaviors of two features of the optimal portfolio in a way similar to that in previous works [7, 13]. In general, we cannot analyze $\psi(n)$ for an arbitrary $n \in \mathbf{R}$ directly, because we cannot differentiate the right-hand side of Eq (27) with respect to the replica number $n$. Thus, as mentioned in the above main manuscript, $n \in \mathbf{N}$ is employed for simplicity. Moreover, it is necessary to assume the optimal solution descriptions of parameters $q_{w a b}, q_{s a b}, \tilde{q}_{w a b}, \tilde{q}_{s a b}, k_{a}$ in Eq (27) appropriately. Specifically, the parameters are assumed to be as follows:

$$
\begin{aligned}
q_{w a b} & =\left\{\begin{array}{ll}
\chi_{w}+q_{w} & a=b \\
q_{w} & a \neq b
\end{array},\right. \\
q_{s a b} & =\left\{\begin{array}{ll}
\chi_{s}+q_{s} & a=b \\
q_{s} & a \neq b
\end{array},\right. \\
\tilde{q}_{w a b} & =\left\{\begin{array}{ll}
\tilde{\chi}_{w}-\tilde{q}_{w} & a=b \\
-\tilde{q}_{w} & a \neq b
\end{array},\right. \\
\tilde{q}_{s a b} & =\left\{\begin{array}{ll}
\tilde{\chi}_{s}-\tilde{q}_{s} & a=b \\
-\tilde{q}_{s} & a \neq b
\end{array},\right. \\
k_{a} & =k .
\end{aligned}
$$

This solution is called the replica symmetry solution and the above assumption is called the replica symmetry ansatz. The effectiveness of the replica symmetry solution and that of the replica symmetry assumption have been verified in the analyses of several problems in statistical learning theory and information theory [11, 12]. In this paper, for the sake of simplicity, the replica symmetry solution is used, where from the expressions of $q_{w}$ and $\chi_{w}$, since there exists symmetry in the replica indices $a, b$, $E_{\vec{w}}\left[w_{i a}^{2}\right]=E_{\vec{w}}\left[w_{i}^{2}\right]$ and $E_{\vec{w}}\left[w_{i a} w_{i b}\right]=E_{\vec{w}}\left[w_{i a}\right] E_{\vec{w}}\left[w_{i b}\right]=$ $\left(E_{\vec{w}}\left[w_{i}\right]\right)^{2},(a \neq b)$ are used. Therefore,

$$
\begin{aligned}
q_{w a a} & =\lim _{N \rightarrow \infty} \frac{1}{N} \sum_{i=1}^{N} E_{\vec{w}}\left[w_{i}^{2}\right], \\
q_{w a b} & =\lim _{N \rightarrow \infty} \frac{1}{N} \sum_{i=1}^{N}\left(E_{\vec{w}}\left[w_{i}\right]\right)^{2},
\end{aligned}
$$


are obtained. Especially, $\chi_{w}=q_{w a a}-q_{w a b}=q_{w a a}-q_{w}$ from $\mathrm{Eq}(56)$ is

$$
\chi_{w}=\lim _{N \rightarrow \infty} \frac{1}{N} \sum_{i=1}^{N}\left(E_{\vec{w}}\left[w_{i}^{2}\right]-\left(E_{\vec{w}}\left[w_{i}\right]\right)^{2}\right) .
$$

Now, from the replica symmetry ansatz,

$$
\begin{aligned}
\psi(n)= & -\frac{n \alpha}{2} \log \left(1+\beta \chi_{s}\right)-\frac{\alpha}{2} \log \left(1+\frac{n \beta q_{s}}{1+\beta \chi_{s}}\right) \\
& +\frac{n}{2}\left(\chi_{w}+q_{w}\right)\left(\tilde{\chi}_{w}-\tilde{q}_{w}\right)-\frac{n(n-1)}{2} q_{w} \tilde{q}_{w} \\
& +\frac{n}{2}\left(\chi_{s}+q_{s}\right)\left(\tilde{\chi}_{s}-\tilde{q}_{s}\right)-\frac{n(n-1)}{2} q_{s} \tilde{q}_{s} \\
& -n k+\frac{1}{2}\left\langle\frac{n k^{2}}{\tilde{\chi}_{w}+s \tilde{\chi}_{s}-n\left(\tilde{q}_{w}+s \tilde{q}_{s}\right)}\right. \\
& \left.-n \log \left(\tilde{\chi}_{w}+s \tilde{\chi}_{s}\right)-\log \left(1-n \frac{\tilde{q}_{w}+s \tilde{q}_{s}}{\tilde{\chi}_{w}+s \tilde{\chi}_{s}}\right)\right\rangle
\end{aligned}
$$

is obtained. From Eq (22) and Eq (64),

$$
\begin{aligned}
\phi= & -\frac{\alpha}{2} \log \left(1+\beta \chi_{s}\right)-\frac{\alpha \beta q_{s}}{2\left(1+\beta \chi_{s}\right)}+\frac{1}{2} q_{w} \tilde{q}_{w} \\
& +\frac{1}{2}\left(\chi_{w}+q_{w}\right)\left(\tilde{\chi}_{w}-\tilde{q}_{w}\right)+\frac{1}{2}\left(\chi_{s}+q_{s}\right)\left(\tilde{\chi}_{s}-\tilde{q}_{s}\right) \\
& +\frac{1}{2} q_{s} \tilde{q}_{s}-k+\frac{1}{2}\left\langle\frac{k^{2}}{\tilde{\chi}_{w}+s \tilde{\chi}_{s}}-\log \left(\tilde{\chi}_{w}+s \tilde{\chi}_{s}\right)\right. \\
& \left.+\frac{\tilde{q}_{w}+s \tilde{q}_{s}}{\tilde{\chi}_{w}+s \tilde{\chi}_{s}}\right\rangle
\end{aligned}
$$

is obtained. Furthermore, we need to determine the extremum of order parameters $\chi_{w}, q_{w}, \chi_{s}, q_{s}$ and their conjugate order parameters $\tilde{\chi}_{w}, \tilde{q}_{w}, \tilde{\chi}_{s}, \tilde{q}_{s}, k$ in the right-hand side of Eq (65). From the extremum of the right-hand side of Eq (65),

$$
\begin{aligned}
\chi_{w} & =\frac{\left\langle s^{-1}\right\rangle}{\beta(\alpha-1)}, \\
q_{w} & =\frac{\left\langle s^{-2}\right\rangle}{\left\langle s^{-1}\right\rangle^{2}}+\frac{1}{\alpha-1}, \\
\tilde{\chi}_{w} & =0 \\
\tilde{q}_{w} & =0, \\
\chi_{s} & =\frac{1}{\beta(\alpha-1)}, \\
q_{s} & =\frac{\alpha}{\left\langle s^{-1}\right\rangle(\alpha-1)}, \\
\tilde{\chi}_{s} & =\beta(\alpha-1), \\
\tilde{q}_{s} & =\frac{\beta^{2}(\alpha-1)}{\left\langle s^{-1}\right\rangle}, \\
k & =\frac{\beta(\alpha-1)}{\left\langle s^{-1}\right\rangle},
\end{aligned}
$$

are obtained. Then, the investment risk per asset of finite temperature $\beta$ is described as follows:

$$
\varepsilon=\frac{\alpha-1}{2}\left(\frac{1}{\left\langle s^{-1}\right\rangle}+\frac{1}{\beta(\alpha-1)}\right) .
$$

Moreover, in the limit of large $\beta$,

$$
\varepsilon=\frac{\alpha-1}{2\left\langle s^{-1}\right\rangle},
$$

is obtained and the concentrated investment level is also assessed in Eq (67).

\section{B. Belief propagation algorithm}

In our previous work [9], an algorithm for solving the portfolio optimization problem defined in Eq (11) and Eq (2) based on belief propagation was proposed. Note that the statistical nature of the return rate was not limited in our previous work, that is, we do not assume that the return rate of each asset is independently and identically distributed. Thus, let the findings in this paper be supported by this belief propagation algorithm. First, this algorithm is expanded as follows:

$$
\begin{aligned}
m_{w i} & =\chi_{w i}\left(h_{w i}+k\right) \\
h_{w i} & =\frac{1}{\sqrt{N}} \sum_{\mu=1}^{p} x_{i \mu} m_{u \mu}+\tilde{\chi}_{w i} m_{w i} \\
\tilde{\chi}_{w i} & =\frac{1}{N} \sum_{\mu=1}^{p} x_{i \mu}^{2} \chi_{u \mu} \\
\chi_{w i} & =\frac{1}{\tilde{\chi}_{w i}} \\
m_{u \mu} & =-\chi_{u \mu} h_{u \mu} \\
h_{u \mu} & =\frac{1}{\sqrt{N}} \sum_{i=1}^{N} x_{i \mu} m_{w i}-\tilde{\chi}_{u \mu} m_{u \mu} \\
\tilde{\chi}_{u \mu} & =\frac{1}{N} \sum_{i=1}^{N} x_{i \mu}^{2} \chi_{w i}, \\
\chi_{u \mu} & =\frac{\beta}{1+\beta \tilde{\chi}_{u \mu}},
\end{aligned}
$$

where $m_{w i}=E_{\vec{w}}\left[w_{i}\right]$ and $\chi_{w i}=E_{\vec{w}}\left[w_{i}^{2}\right]-\left(E_{\vec{w}}\left[w_{i}\right]\right)^{2}$ are already used in [9] and it is indicated that the solution of these simultaneous equations is consistent with the optimal portfolio in the previous work. In practice, from $\mathrm{Eq}(77)$ to $\mathrm{Eq}(84)$,

$$
k=\frac{\beta}{N} \sum_{j=1}^{N} \sum_{\mu=1}^{p} x_{i \mu} x_{j \mu} m_{w j},
$$

is obtained. This can be expressed in the following vector-matrix form:

$$
k \vec{e}=\beta J \vec{m}_{w},
$$


where matrix $J=\left\{J_{i j}\right\} \in \mathcal{M}_{N \times N}$ is given in Eq (4) and $\vec{m}_{w}=\left(m_{w 1}, m_{w 2}, \cdots, m_{w N}\right)^{\mathrm{T}} \in \mathbf{R}^{N}$. This implies $\vec{m}_{w}=\frac{k}{\beta} J^{-1} \vec{e}$. In addition, since $\vec{m}_{w}$ satisfies Eq (2), then $\frac{k}{\beta}=\frac{N}{\vec{e}^{T} J^{-1} \vec{e}}$ is obtained. If we substitute this into $\mathrm{Eq}(\underline{86})$, then

$$
\vec{m}_{w}=\frac{N J^{-1} \vec{e}}{\vec{e}^{T} J^{-1} \vec{e}}
$$

is obtained, which can easily be shown to be consistent with Eq (3).

Next, assume that the number of assets $N$ is sufficiently large (but not infinity). Since return rates $x_{i \mu}$ are independently distributed with mean and variance 0 and $s_{i}$, respectively, and we assume that one can replace $\chi_{u \mu}$ and $\tilde{\chi}_{u \mu}$ by $\chi_{u}$ and $\tilde{\chi}_{u}$, respectively. Then,

$$
\frac{1}{p} \sum_{\mu=1}^{p} x_{i \mu}^{2} \simeq E_{X}\left[x_{i \mu}^{2}\right]=s_{i}
$$

is approximately estimated. From this,

$$
\begin{aligned}
\tilde{\chi}_{w i} & =\frac{p}{N} s_{i} \chi_{u}=\alpha s_{i} \chi_{u}, \\
\chi_{w i} & =\frac{1}{\alpha s_{i} \chi_{u}}, \\
\tilde{\chi}_{u} & =\frac{1}{N} \sum_{i=1}^{N} s_{i} \chi_{w i}=\frac{1}{\alpha \chi_{u}}, \\
\chi_{u} & =\frac{\beta}{1+\frac{\beta}{\alpha \chi_{u}}}=\beta\left(1-\frac{1}{\alpha}\right),
\end{aligned}
$$

are obtained. From these equations, the expectation of the variance of $w_{i}$ is

$$
\chi_{w}=\frac{1}{N} \sum_{i=1}^{N} \chi_{w i}=\frac{\left\langle s^{-1}\right\rangle}{\beta(\alpha-1)} .
$$

This is consistent with Eq (66).
[1] Z. Bodie, A. Kane, A. J. Marcus, Investments (McGrawHill Education, 2014).

[2] D. G. Luenberger, Investment science (Oxford University Press, 1997).

[3] H. Markowitz, J. Fin. 7, 77 (1952).

[4] H. Markowitz, Portfolio selection: efficient diversification of investments (J. Wiley and Sons, New York., 1959).

[5] H. Konno, H. Yamazaki, Man. Sci. 37, 519 (1991).

[6] R. T. Rockafellar, S. Uryasev, J. Risk, 2, 21. (2000).

[7] S. Ciliberti, M. Mézard, Euro. Phys. J. B, 27, 175 (2007).
[8] S. Pafka, I. Kondor, Euro. Phys. J. B, 27, 277 (2002).

[9] T. Shinzato, M. Yasuda, PLoS One, 10, e0134968 (2015).

[10] T. Shinzato, PLoS One, 10, e0133846 (2015).

[11] T. Shinzato, Y. Kabashima, J. Phys. A. 41, 324013 (2008).

[12] Y. Kabashima, D. Saad, J. Phys. A. 37, R1 (2004).

[13] H. Nishimori, Statistical physics of spin glasses and information processing (Oxford University Press, 2001). 